\title{
Automated breast ultrasound (ABUS) as a screening tool: initial experience
}

\author{
Amera Abd Elsalam Mostafa', Mohamed Adel Eltomey², Ashraf Mohammed Elaggan² and Amel A. Hashish ${ }^{3}$
}

\begin{abstract}
Background: Breast cancer is a major health problem, being the most common cancer in women. Early detection of breast cancer aims to the reduction of mortality and morbidity rates. Conventional screening methods include mammography and ultrasonography; however, both modalities have their limitations. Automated breast ultrasound (ABUS) is a recent technological advancement in the field of breast imaging having the benefit of standardization of the scans and lack of operator dependence as in conventional handheld ultrasound scans. The aim of this work was to report our initial experience of the added value of ABUS as a breast screening tool. The study included 200 patients who had screening mammograms, ultrasound, and ABUS.

Results: A significant difference was found between the number of lesions detected by ABUS and conventional ultrasound. A significant difference was found between lesions detected by ABUS and mammography which was most evident in patients with dense breasts.

Conclusions: ABUS is a valuable tool in the screening of the breast with improved lesion detection, especially in patients with dense breasts.
\end{abstract}

Keywords: ABUS, Automated breast ultrasound, Breast screening, Ultrasound

\section{Background}

Breast cancer is a major worldwide health problem as it is the most common cancer in women. It accounts for $22.9 \%$ of all new female cancers worldwide and in Egypt for $37.7 \%$ of total female cancers and $29.1 \%$ of cancerrelated death [1].

The aim of early detection of breast cancer is to reduce the morbidity and mortality rates [2]. Mammography has been established as the imaging modality for screening and early detection of breast cancer [3]. The major disadvantage of mammography however is the relatively low sensitivity and specificity, especially in women with dense breasts, secondary to the low contrast between the density of tumor tissue and the surrounding breast tissue [4].

Breast ultrasound combined with mammography has proven to improve tumor detection rates specifically in women with dense breasts (ACR C and D types). Conventional breast ultrasound is operator-

\footnotetext{
* Correspondence: mohamed.eltomey@med.tanta.edu.eg

${ }^{2}$ Radiology and Medical Imaging Department, Faculty of Medicine, Tanta

University, Tanta, Egypt

Full list of author information is available at the end of the article
}

dependent which implies that its results are affected by the operator skill and experience with consequent loss of standardization [5].

Automated breast ultrasound (ABUS) is a technological advancement in the field of ultrasound imaging that was developed to overcome the issue of operator dependence of conventional US scanning by standardization of the images acquired. This is achieved by volumetric acquisition of multiple US images of the breast that can be stored and post processed, i.e., 3D and multiplaner reformatting, for later reading and evaluation [5, 6]. Despite automated breast ultrasound (ABUS) has been around for more than a decade yet earlier systems provided inadequate image quality for proper interpretation. Recent developments in ABUS systems with the use of high-frequency transducers allowed for a reproduction of images with a superior quality in a short time as well [7].

\section{Methods}

The aim of this work is to study the added value of ABUS in the screening of women with suspected breast masses compared to conventional mammography and hand-held ultrasound. 
The study included 200 female patients during the period from February 2017 to January 2018.

Inclusion criteria were any female in the child-bearing age complaining of breast pain or lump and those with a positive family history of breast cancer. Exclusion criteria included patients with a known diagnosis of breast cancer under treatment and those with history of mastectomy or previous interventions for breast lesions.

All patients were subjected to a full clinical examination, 80 patients had biopsy and histopathological analysis based on imaging findings.

Routine screening mammography using craniocaudal and mediolateral oblique views for both breasts was performed in 108 patients as those below the age of 30 were screened by ultrasound only as recommended by the international guidelines [8]. Mammography was performed using Senographe Essential (GE Healthcare, USA) full-field digital mammography (FFDM) machine. Mammograms were reviewed for the breast density, presence of masses with detailed analysis of its site, type, number, border, calcifications, and size.

All 200 patients had a hand-held (conventional) ultrasound (HHUS) scan and automated breast ultrasound (ABUS) for both breasts. Hand-held ultrasound was performed on a Toshiba Nemio XG Ultrasound machine (Toshiba Medical Systems, Japan) using a 7-11 MHz linear transducer. The technique performed for HHUS was done after exposure of the breasts with the patient lying supine and her ipsilateral hand raised above the head. The ultrasound probe was oriented perpendicular to the chest wall. Radial scanning technique, in a clockwise fashion, using the nipple as a center point was followed. Scanning of each breast quadrant in the sagittal and transverse planes was also performed and the examination time took about $20 \mathrm{~min}$.

Automated breast ultrasound (ABUS) was performed using the Invenia ABUS machine (GE Healthcare, USA) which is equipped with $14 \mathrm{MHz}$ linear transducer. The scan was performed with the patient in a supine position, a wedge is placed under the back of the patient on the side to be examined; this allows for flattening the breast for improved contact with the probe. After exposure of the breast, application of an adequate amount of coupling gel over the entire surface of the breast is done with an extra amount applied to the nipple-areolar complex to insure proper scanning and avoid scanning artifacts. The transducer was applied to the breast using mild compression to avoid motion artifacts. Scanning was performed in three standard views (coronal, longitudinal, and transverse views) to allow for adequate coverage of the entire breast tissue. Using the nipple as a centering point, the anteroposterior (AP) position is first taken. Lateral position is taken by angling the probe from the axilla towards the sternum and for medial position the probe is angled from the sternum towards the axilla. The scans are initially reviewed on the machine monitor for quality assurance of the acquired scan. The scan time for each acquisition was $1 \mathrm{~min}$ and volumetric data obtained were automatically transferred to the Invenia ABUS workstation for post processing including axial, coronal, and sagittal reformats and analysis. The entire examination time was approximately 15 min for both sides.

Ultrasound studies were reviewed for the presence or absence of lesions, location, size, number, outline and contour (regular or irregular), margin or borders (micro or macro lobulations), echogenicity compared to the breast fat, and internal echopattern (homogenous or heterogeneous)

The study researchers reviewed the mammograms, conventional ultrasound, and ABUS findings in conjunction since it was their initial experience with ABUS. The researchers had a combined 10 years experience in breast imaging in between them.

\section{Statistical analysis}

Descriptive statistics were used in terms of frequencies (number of cases) and percentages when appropriate. Accuracy was represented using the terms sensitivity, specificity, positive predictive value, and negative predictive value.

Analytic statistics using $t$ test and chi-square tests were used to compare the clinical and imaging findings obtained using pathological diagnosis as the gold standard of the detected masses.

\section{Results}

The study included 200 female patients aged between 19 and 61 years (mean 35.44 \pm SD 10.83). The most common patient complaint was pain in 140 patients representing $70 \%$ of the patient population and a lump in 32 patients representing $16 \%$.

All patients were examined by ABUS and HHUS, but only 108 patients above the age of 30 years had mammograms performed. The number of patients with dense breasts, ACR C, and D, were 28 and 48, respectively, with a total of 76 , representing $70.3 \%$ of the patients screened by mammography.

In this study, 120 patients were found to have negative imaging and pathological findings. Eighty patients had different breast pathologies, 48 showed benign findings and 32 showed malignant disease. The most common benign finding was fibroadneoma in 36 patients, and the most common malignant finding was Invasive ductal carcinoma in 22 patients. Fifty patients showed a single lesion and 30 showed multiple lesions with 20 of them showing lesions in both breasts. 
Using ABUS lesions were detected in 76 patients out of 80 while by using HHUS the lesions were detected in 66 out of 80 patients. There was a statistically significant difference between the number of patients with lesions detected by ABUS and HHUS with $p$ value of 0.012 . There was no statistically significant difference between ABUS and HHUS in the detection of multiple lesions in the same patients with a $p$ value of 0.16 (Figs. 1 and 2). The sensitivity, specificity, positive predictive, and negative predictive values for ABUS and HHUS are shown in Table 1.

Using mammography alone, lesions were detected in 24 out of 40 patients with positive findings, and addition of ABUS to mammography increased this number as lesions were detected in 38 out of 40 patients. A statistically significant difference was found with $p$ value $=$ 0.0001 (Table 2).

The added value of ABUS to mammography in detection of breast lesions was most noted in patients with dense and extremely dense breasts (ACR C and D) as a statistically significant difference was found with $p$ value $=0.0001$. Using mammography alone, 20 out of 36 lesions were detected while with the addition of ABUS 34 out of 36 lesions were detected (Fig. 3; Table 3)

A statistically significant difference was found between ABUS and HHUS in the detection of lesions smaller than $5 \mathrm{~mm}$, when compared to HHUS. The number of lesions detected by ABUS smaller than $5 \mathrm{~mm}$ was 10 compared to 4 by HHUS with a $p$ value of 0.002 .

\section{Discussion}

Automated breast ultrasound machines are considered as a recent addition in breast screening tools intended to overcome some of the limitations of conventional hand held ultrasound scanning [9]. The ultimate goal of a successful mammographic screening program is the early detection of breast cancer [10].

In this study, there was statistically significant increase in the number of cases with lesions detected by ABUS compared to HHUS with increase in the accuracy $98 \%$ compared to $93 \%$, sensitivity $95 \%$ compared to $82 \%$, $100 \%$ sensitivity and negative predictive value $96.8 \%$ compared to $89.6 \%$. The findings in this study are in agreement with those reported by Choi et al. [11] who
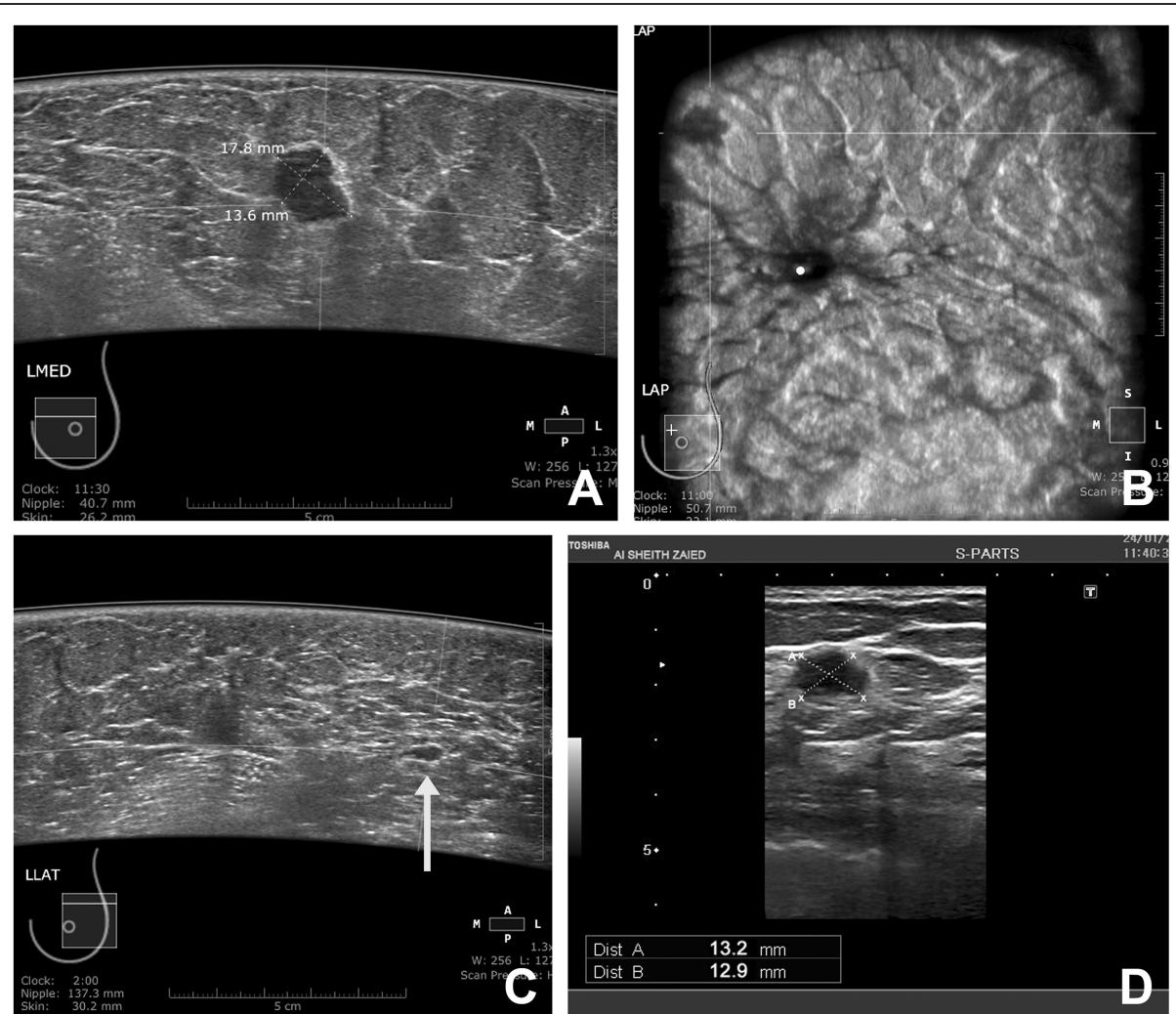

Fig. 1 Female patient 26 years old with histopathological diagnosis of an invasive ductal carcinoma type II. a, b Axial and coronal reformatted ABUS images shows a well-defined hypoechoic mass showing irregular outline in the left breast at its upper inner quadrant. The lesion measured $13.6 \times 17.8$ mm. c Axial reformatted ABUS image showing another well-defined smaller hypoechoic lesion in the upper outer quadrant (arrow) of the left breast, which was not seen by conventional HHUS. The smaller lesion measured $3 \times 4 \mathrm{~mm}$. $\mathbf{d}$ Corresponding HHUS of the larger left breast mass lesion, the lesion is well-defined, hypoechoic, having lobulated outlines and measuring $13.2 \times 12.9 \mathrm{~mm}$. The lesion was graded as BIRADS IV 

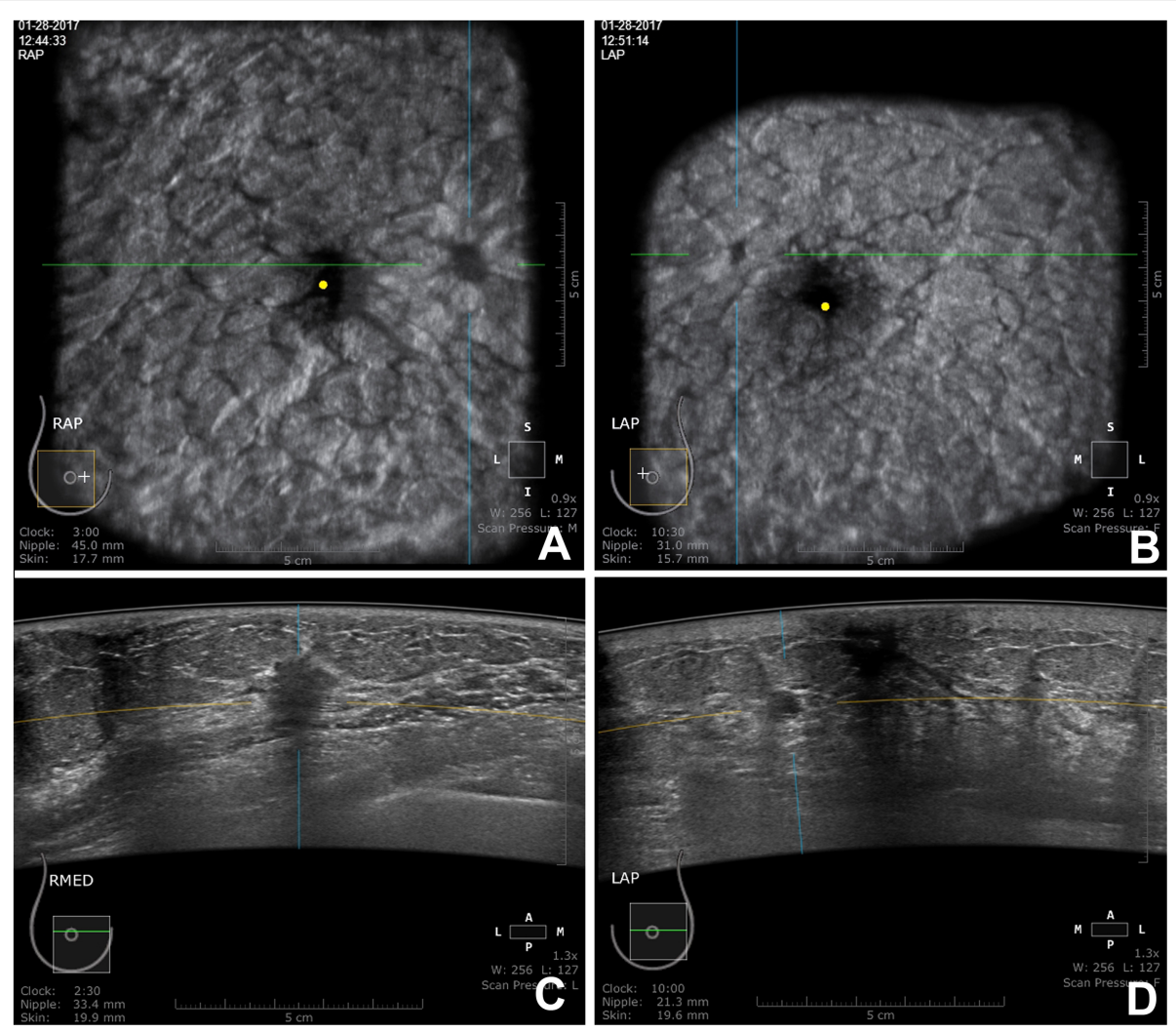

Fig. 2 Female patient 24 years old with histopathological diagnosis of an invasive ductal carcinoma type II. a Coronal reformatted ABUS images of the right breast showing a well-defined hypoechoic lesion in its upper inner quadrant. $\mathbf{b}$ Coronal reformatted ABUS images of the left breast showing a smaller well defined hypoechoic lesion in its upper inner quadrant. c Axial ABUS image of the lesion in the right breast showing a well-defined hypoechoiec lesion showing irregularity of its border with posterior acoustic shadowing the lesion was graded as a BI-RADS IV lesion. $\mathbf{d}$ Axial ABUS image of the lesion in the left breast showing a small rounded hypoechoiec lesion with well-defined border and regular outlines. This lesion was missed on HHUS

reported a statistically significant difference between lesion detection by ABUS compared to HHUS and their diagnostic accuracy, sensitivity, and specificity were $97.70 \%, 77.78 \%$, and $97.79 \%$ respectively. Vourtsis et al. [12] reported in their study that ABUS was comparable to HHUS is lesion detection; however, it outperformed HHUS in detection of architectural distortion, though they did not report sensitivity or specificity of each technique.

Table 1 The comparison between ABUS and HHUS in lesion detection as regards the number of patients. $t$ test was used to compare in between with $p$ value $<0.05$ considered significant

\begin{tabular}{llll}
\hline & ABUS & HHUS & $p$ \\
\hline Lesions detected & $76 / 80$ & $66 / 80$ & 0.012 \\
Accuracy & $98.0 \%$ & $93.0 \%$ & 0.016 \\
Sensitivity & $95.0 \%$ & 82.5 & 0.012 \\
Specificity & $100.0 \%$ & $100.0 \%$ & - \\
PPV & $100.0 \%$ & $100.0 \%$ & - \\
NPV & $96.8 \%$ & 89.6 & 0.023 \\
\hline
\end{tabular}

A statistically significant increase of the number of cases with lesions detected by the addition of ABUS to mammography and the use of mammography alone was found in this study, 38 out of 40 compared to 24 out of 40 lesions with $p$ value $<0.001$. The previous findings were most evident in the 36 patients with dense breasts (ACR $\mathrm{C}$ and $\mathrm{D}$ ) where lesions were detected in 20 patients by the use of mammography alone, and 34 patients detected with the addition of ABUS. The sensitivity of lesion

Table 2 The comparison between the numbers of patients with lesion detected by mammography alone and with the addition of ABUS. $t$ test was used to compare in between with $p$ value $<0.05$ considered significant

\begin{tabular}{llll}
\hline & Mammography & Mammography + ABUS & $p$ \\
\hline Lesions detected & $24 / 40$ & $38 / 40$ & 0.001 \\
Accuracy & $85.19 \%$ & $98.15 \%$ & 0.0005 \\
Sensitivity & $60 \%$ & $95 \%$ & 0.012 \\
Specificity & $100.0 \%$ & $100.0 \%$ & - \\
PPV & $100.0 \%$ & $100.0 \%$ & - \\
NPV & $80.95 \%$ & $97.14 \%$ & 0.023 \\
\hline
\end{tabular}




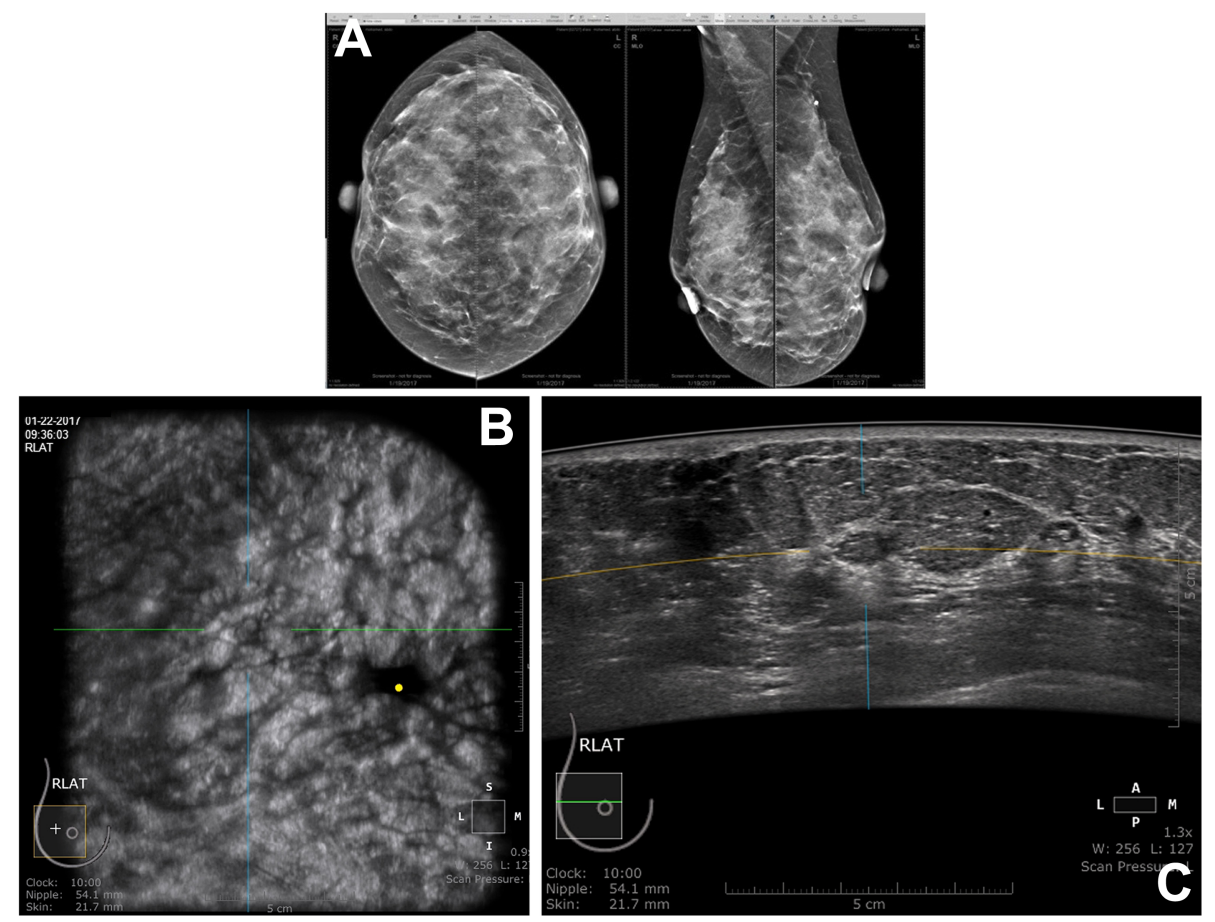

Fig. 3 Female patient 31 years old with histopathological diagnosis of fibroadenoma. a Mammogram (cranio-caudal and mediolateral oblique views of both breasts) showing dense breast (ACR D) with no obvious lesions or calcifications. $\mathbf{b}$ Coronal reformatted ABUS image showing a small hypoechoic lesion in the upper outer quadrant of the right breast. c Axial reformatted ABUS image showing the small well-defined hypoechoic lesion which had a fine lobulated outline. The lesion was graded BI-RADS III

detection by mammography alone in our study was $60 \%$ and $95 \%$ when ABUS was combined with mammography. Our results are in agreement with the similar results showing an increase of the sensitivity of cancer detection by $36 \%$ using ABUS in combination with mammography reported by Wilczek et al. [13] and Kelly et al [14]. The previous findings are also in agreement with Giger et al. [15] who stated a statistically significant increase in the detection rate of cancers with the use of ABUS combined with FFDM in patients with extremely dense breasts. Brem et al. [16] stated an increased rate of cancer detection with the addition of ABUS to screening mammography in patients with dense breasts; however, there was an increase in the number of false positive results as well.

In this study, there was a statistically significant increase in the detection of lesions smaller than $5 \mathrm{~mm}$ by ABUS when compared to HHUS. These findings are in agreement with those reported by Kelly et al. [14] who reported a significant increase in the number of detected small invasive cancers measuring less than $20 \mathrm{~mm}$ in size when adding ABUS to mammography.

Limitations of this study include the small number of patients, the relative bias in case selection as the researchers were still along the learning phase of this technique during the study, and that ABUS is a recently introduced imaging modality in Egypt with limited number of machines; to the best of our knowledge, two machines were installed at the time this study was conducted. Further studies incorporating this modality with the national screening program would definitely provide more information regarding the efficacy of the technique and the cost-benefit of its use on routine basis.

Table 3 Comparison between ABUS and mammography in the detection of lesions in patients with dense breasts ACR C and ACR D. $t$ test was used to compare between the results with $p$ value $<0.05$ considered significant

\begin{tabular}{|c|c|c|c|c|}
\hline ACR breast density & $\begin{array}{l}\text { Number of lesions } \\
\text { detected }\end{array}$ & $\begin{array}{l}\text { Lesions detected by } \\
\text { mammography alone }\end{array}$ & $\begin{array}{l}\text { Lesions detected by } \\
\text { mammography }+\mathrm{ABUS}\end{array}$ & $p$ value \\
\hline C (dense) & 8 & 4 & 6 & 0.13 \\
\hline D (extremely dense) & 28 & 16 & 28 & 0.0005 \\
\hline$C$ and D & 36 & 20 & 34 & 0.0001 \\
\hline
\end{tabular}




\section{Conclusions}

Automated breast ultrasound (ABUS) is a technological advancement in breast imaging and screening with the benefits of standardization of the scans, better detection of small lesions especially in patients with dense breasts and improved scan times. The main drawback in our opinion is the relatively high cost of the machine, compared to conventional machines, limiting its spread.

\section{Abbreviations}

ABUS: Automated breast ultrasound; CC: Craniocaudal; FFDM: Full-field digital mammography; HHUS: Hand-held ultrasound; MLO: Mediolateral oblique

\section{Acknowledgements}

Not applicable

\section{Authors' contributions}

All authors have appraised the article and actively contributed to the work. AM conceived the idea of research, helped in the scanning of the patients, and collected the data. ME wrote the manuscript and contributed to the statistical analysis and revision of the manuscript. AE helped revise the images and manuscript. AH contributed to the correlation of clinical and imaging data and overall revision of the manuscript. All authors read and approved the final manuscript.

\section{Funding}

The authors declare that the study was self-funded, and no funding was obtained from the manufacturers of the machines used, from any of its representatives, or from any other organization.

\section{Availability of data and materials}

The data sets generated and/or analyzed in this study are available through the corresponding author on reasonable request.

\section{Ethics approval and consent to participate}

This study was approved by the Research Ethics Committee of the Faculty of Medicine at Tanta University in Egypt on February 2017; Reference Number of approval: 31366/02/17. All patients included in this study gave written informed consent to participate in this research.

\section{Consent for publication}

All patients included in this research gave written informed consent to publish the data contained within this study. The authors grant the publisher the consent for publication of this work.

\section{Competing interests}

The authors declare that they have no competing interests.

\section{Author details}

${ }^{1}$ Radiology Department, El-Sheikh Zayed Al Nahyan Hospital, Cairo, Egypt ${ }^{2}$ Radiology and Medical Imaging Department, Faculty of Medicine, Tanta University, Tanta, Egypt. ${ }^{3}$ Surgery Department, Faculty of Medicine, Tanta University, Tanta, Egypt.

Received: 16 July 2019 Accepted: 13 August 2019

Published online: 02 October 2019

\section{References}

1. Zeeneldin AA, Ramadan M, Gaber AA, Taha FM (2013) Clinico-pathological features of breast carcinoma in elderly Egyptian patients: a comparison with the non-elderly using population-based data. J Egypt Natl Cancer Inst. 25(1):5-11

2. Monticciolo DL, Newell MS, Moy L, Niell B, Monsees B, Sickles EA (2018) Breast cancer screening in women at higher-than-average risk: recommendations from the ACR. J Am Coll Radiol. 15(3):408-414

3. van den Biggelaar FJ, Kessels AG, van Engelshoven JM, Flobbe K (2009) Strategies for digital mammography interpretation in a clinical patient population. Int J Cancer. 125(12):2923-2929

4. Fallenberg EM, Dromain C, Diekmann F, Engelken F, Krohn M, Singh JM et al (2014) Contrast-enhanced spectral mammography versus MRI: initial results in the detection of breast cancer and assessment of tumour size. Eur Radiol. 24(1):256-264

5. Xiao Y, Zhou Q, Chen Z (2015) Automated breast volume scanning versus conventional ultrasound in breast cancer screening. Acad Radiol. 22(3):387-399

6. Zanotel M, Bednarova I, Londero V, Linda A, Lorenzon M, Girometti R et al (2018) Automated breast ultrasound: basic principles and emerging clinical applications. La Radiologia medica. 123(1):1-12

7. Shin HJ, Kim HH, Cha JH (2015) Current status of automated breast ultrasonography. Ultrasonography. 34(3):165-172

8. Mainiero MB, Moy L, Baron P, Didwania AD, diFlorio RM, Green ED et al (2017) ACR appropriateness criteria breast cancer screening. J Am Coll Radiol. 14(11):S383-SS90

9. van Zelst JCM, Mann RM (2018) Automated three-dimensional breast US for screening: technique, artifacts, and lesion characterization. Radiographics 38 (3):663-683

10. Saadatmand S, Bretveld R, Siesling S, Tilanus-Linthorst MMA (2015) Influence of tumour stage at breast cancer detection on survival in modern times: population based study in 173797 patients. BMJ. 351:h4901

11. Choi WJ, Cha JH, Kim HH, Shin HJ, Kim H, Chae EY et al (2014) Comparison of automated breast volume scanning and hand-held ultrasound in the detection of breast cancer: an analysis of 5,566 patient evaluations. Asian Pac J Cancer Prev. 15(21):9101-9105

12. Vourtsis A, Kachulis A (2108) The performance of $3 D$ ABUS versus HHUS in the visualisation and BI-RADS characterisation of breast lesions in a large cohort of 1,886 women. Eur Radiol. 28(2):592-601

13. Wilczek B, Wilczek HE, Rasouliyan L, Leifland K (2016) Adding 3D automated breast ultrasound to mammography screening in women with heterogeneously and extremely dense breasts: Report from a hospitalbased, high-volume, single-center breast cancer screening program. Eur J Radiol. 85(9):1554-1563

14. Kelly KM, Dean J, Comulada WS, Lee SJ (2010) Breast cancer detection using automated whole breast ultrasound and mammography in radiographically dense breasts. Eur Radiol. 20(3):734-742

15. Giger ML, Inciardi MF, Edwards A, Papaioannou J, Drukker K, Jiang $Y$ et al (2016) Automated breast ultrasound in breast cancer screening of women with dense breasts: reader study of mammography-negative and mammography-positive cancers. AJR Am J Roentgenol. 206(6):1341-1350

16. Brem RF, Tabár L, Duffy SW, Inciardi MF, Guingrich JA, Hashimoto BE et al (2015) Assessing improvement in detection of breast cancer with threedimensional automated breast US in women with dense breast tissue: the Somolnsight study. Radiology. 274(3):663-673

\section{Publisher's Note}

Springer Nature remains neutral with regard to jurisdictional claims in published maps and institutional affiliations.

\section{Submit your manuscript to a SpringerOpen ${ }^{\bullet}$ journal and benefit from:}

- Convenient online submission

- Rigorous peer review

- Open access: articles freely available online

- High visibility within the field

- Retaining the copyright to your article

Submit your next manuscript at $\boldsymbol{\nabla}$ springeropen.com 